\title{
Analgesic efficacy of ketorolac and morphine in neonatal rats
}

\author{
Anju Gupta ${ }^{\mathrm{a}}$, Jainxin Cheng ${ }^{\mathrm{b}}$, Shaoning Wang ${ }^{\mathrm{b}}$, Gordon A. Barr ${ }^{\mathrm{b}, \mathrm{c}, *}$ \\ ${ }^{a}$ Division of Neonatology, Department of Pediatrics, Columbia-Presbyterian Medical Center, Babies Hospital, 3959 Broadway, Room 1201, New York, NY \\ 10032, USA \\ ${ }^{\mathrm{b}}$ Department of Developmental Psychobiology, New York State Psychiatric Institute, 1051 Riverside Drive, New York, NY 10032, USA \\ ${ }^{\mathrm{c}}$ Department of Psychology, Hunter College, 695 Park Avenue, New York, NY 10032, USA
}

Received 13 April 2000; received in revised form 11 October 2000; accepted 2 November 2000

\begin{abstract}
Ketorolac is a potent nonsteroidal antiinflammatory drug (NSAID). In adult humans and animals, its analgesic efficacy can be comparable to opiates. However, it has not been studied in neonatal animals. We conducted a blinded, controlled study comparing the effects of ketorolac and morphine in neonatal rats using the formalin model. Animals were given intraperitoneal (ip) injections of ketorolac or morphine at 3 or 21 days of age. Ketorolac had an analgesic and antiinflammatory effect in 21-day-old pups, but not in the 3day-olds. Morphine had a significant analgesic, but no antiinflammatory effect at both ages. These results indicate that ketorolac is an effective analgesic agent in preweaning, but not neonatal rats. Opiates may be more appropriate analgesics in neonates. (C) 2001 Elsevier Science Inc. All rights reserved.
\end{abstract}

Keywords: Ketorolac; Morphine; Neonatal rats; Formalin

\section{Background}

Ketorolac is one of the most potent nonsteroidal antiinflammatory (NSAID) drugs available for clinical use. It can be administered orally, parenterally, and topically (for ocular inflammation). Its effects are mainly peripheral due to nonselective inhibition of cyclo-oxygenase and subsequent reduction of prostaglandin production (especially prostaglandin E2) (Zhang et al., 1997). Data from animal studies also suggest that ketorolac may have a central modulatory effect on opioid pharmacology (Maves et al., 1994).

In human adults, ketorolac has been extensively evaluated in the relief of postoperative pain and other acute pain states. It has similar analgesic efficacy as morphine and meperidine in relieving postoperative pain, and is tolerated well with few side effects (renal, gastrointestinal, platelet function) (Gillis and Brogden, 1997). In the emergency department, in patients with renal colic (Larkin et al., 1999), migraine headache, musculoskeletal pain, or sickle crisis, ketorolac reduces pain as effectively as opiates (Gillis

\footnotetext{
* Corresponding author. Department of Psychology, Hunter College, 695 Park Avenue, New York, NY 10032, USA. Tel.: +1-212-7725610; fax: +1-212-7724477.

E-mail address: gbarr@shiva.hunter.cuny.edu (G.A. Barr).
}

and Brogden, 1997). Ketorolac also has a synergistic effect with morphine and fentanyl and reduces the need for opiates in the postoperative period in both human adults (Picard et al., 1997) and children (Chiaretti et al., 1997). The combination of ketorolac and opiates allows the use of subtherapeutic doses of both drugs and reduction in their side effects. In the pediatric population, ketorolac has mostly been used in children over the age of 2 years. Its analgesic efficacy is comparable to opiates in strabismus surgery (Shende and Das, 1999), bladder surgery (Gonzalez and Smith, 1998), tonsillectomy (Mather and Peutrell, 1995), and dental surgery (Purday et al., 1996). There is no reported experience with its use in human neonates.

When tested in animal models, ketorolac is an effective analgesic, antiinflammatory and antipyretic drug in adult rats (Rooks, 1990; Jett et al., 1999) and mice (Uphouse et al., 1993). In rats, its analgesic efficacy is $300-500$ times that of aspirin (Rooks et al., 1985). In adult rat models of inflammatory pain using intraarticular injection of uric acid, ketorolac is as effective as morphine and more potent than aspirin in relieving pain. (Granados-Soto et al., 1993). For adult animals, the range of effective doses and types of inflammatory models reported in literature is wide, from 1 $\mathrm{mg} / \mathrm{kg}$ in rats with uric-acid-induced arthritis (GranadosSoto et al., 1993) up to $200 \mathrm{mg} / \mathrm{kg}$ in rats injected with 
formalin (Bustamente and Paeile, 1993). In the rat formalin test, two phases of nociceptive behavior are seen following formalin injection into the subcutaneous space, phase $1(0-$ $10 \mathrm{~min}$ ) is pain produced by direct stimulation and phase 2 $(10 \mathrm{~min}-1 \mathrm{~h})$ is pain due to sensitization of primary afferents and the spinal cord. In adult rats, ketorolac reduced phase 2 of inflammation-induced pain, but had no effect on phase 1 pain. There are no data on the analgesic efficacy of ketorolac in animal neonatal models. Therefore, we studied its analgesic and antiinflammatory effects in neonatal and infant rats using the formalin model to produce acute pain and inflammation. A behavioral scoring system was used to assess pain, paw diameter was measured to assess inflammation, and Fos staining of spinal cord sections was used to assess neural response to pain. Our hypothesis was that ketorolac would be an effective analgesic and antiinflammatory agent in neonatal and preweaning rats, with analgesic efficacy comparable to that of morphine.

\section{Materials and methods}

This was a randomized, blinded, controlled study using a total of 136 rat pups. All experiments were approved by New York State Psychiatric Institute IACUCs and followed Ethical Guidelines of the Society for Neuroscience and the International Society for Developmental Psychobiology.

\subsection{Experimental animals}

Subjects were awake Long-Evans hooded rat pups (3 and 21 days old). They were born and bred in our animal facility. The pups were housed with their mothers in plastic cages measuring $40 \times 20 \times 24 \mathrm{~cm}$ with bedding, food and water available ad libitum. The cages were in a colony room maintained at $24^{\circ} \mathrm{C}$ with a 12 -h light-dark cycle (lights on at 08:00 hours). Cages were checked twice a day at 09:00 and 18:00 hours and pups found at either time were designated 0 days of age. Pups were separated from their mothers immediately prior to the experiment and were kept warm using incubators/heating pads.

\subsection{Drug preparation and administration}

The drugs used in this study were ketorolac (15-45 $\mathrm{mg} / \mathrm{kg}$ ip; Toradol, Roche, Nutley, NJ) and morphine sulfate $(0.1-2 \mathrm{mg} / \mathrm{kg}$ ip; Merck, Rahway, NJ). Drugs were dissolved in $0.9 \%$ saline solution and injected in volumes of $1 \mathrm{cc} / 100 \mathrm{~g}$ body weight $1 \mathrm{~h}$ prior to the formalin injection for ketorolac and half an hour prior to formalin injection for morphine. Three-day-old rats were given intraperitoneal (ip) injections of either saline or ketorolac 15,35 , and $45 \mathrm{mg} / \mathrm{kg}$, or morphine $0.1,0.3$, and $1.0 \mathrm{mg} / \mathrm{kg}$. Older rats were given intraperitoneal injections of either saline or ketorolac 15, 35, and 45 $\mathrm{mg} / \mathrm{kg}$, or morphine $0.2,0.6$, and $2.0 \mathrm{mg} / \mathrm{kg}$. All doses were given within litter. Lower doses were used in the younger animals, since morphine is more potent in younger animals.

\subsection{Formalin}

Ten microliters of $2 \%$ formalin was injected into the subcutaneous space of the plantar aspect of the left hindpaw. Pain behavior was recorded for a period of $60 \mathrm{~min}$, starting immediately after the formalin injection. The pain behavior was recorded at 1-min intervals, using a four-point scale as described below:

Score 1 - paw resting on surface (similar to uninjected paw).

Score 2 - paw lifted up above the surface.

Score 3 - paw shaken.

Score 4 - paw licked.

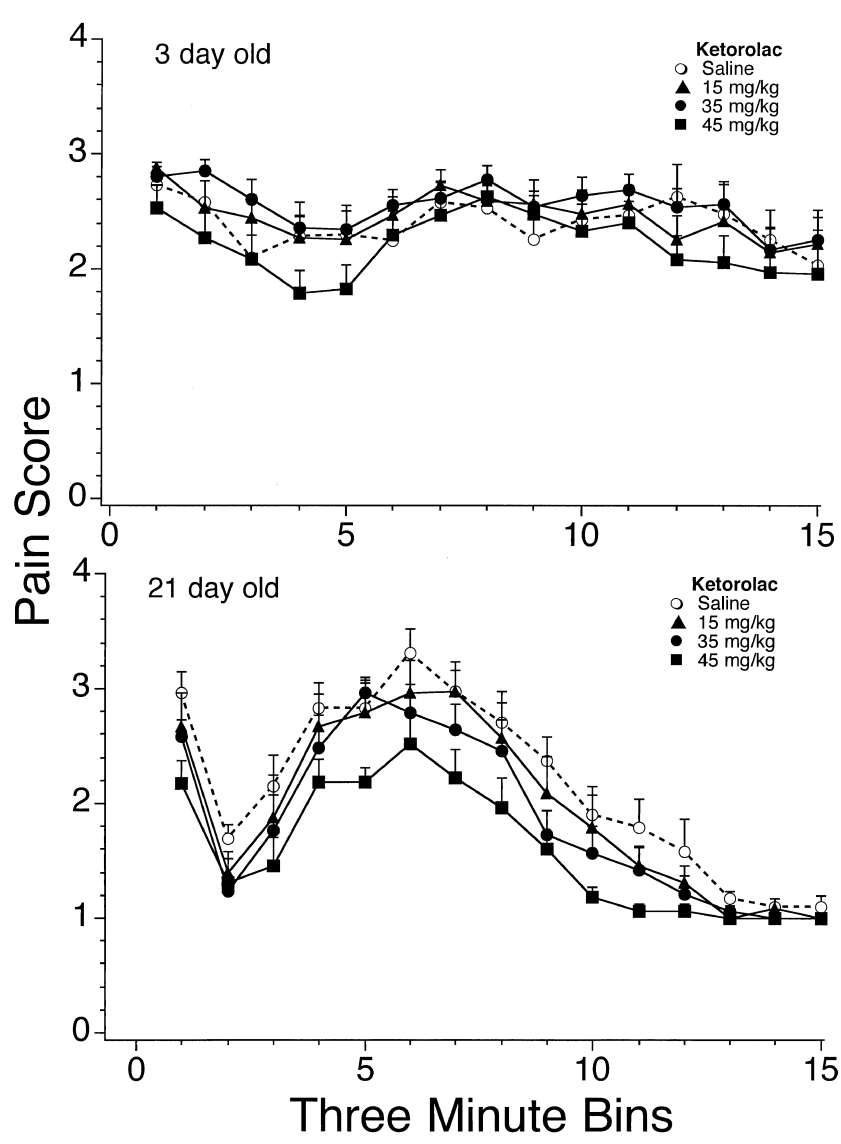

Fig. 1. Mean ( \pm S.E.M.) nociceptive scores across the first 15 three-minute bins in 3- and 21-day-old rat pups that received intraperitoneal saline, 15, 35 , or $45 \mathrm{mg} / \mathrm{kg}$ of ketorolac prior to an intraplantar injection of $2 \%$ formalin $(10 \mu \mathrm{l})$. The older animals treated with ketorolac showed a reduction in nociceptive scores during phases 1 and 2 and a dose-response effect was seen $(P<.01$ with $45 \mathrm{mg} / \mathrm{kg}$ ketorolac). No significant effect was seen in younger pups. 


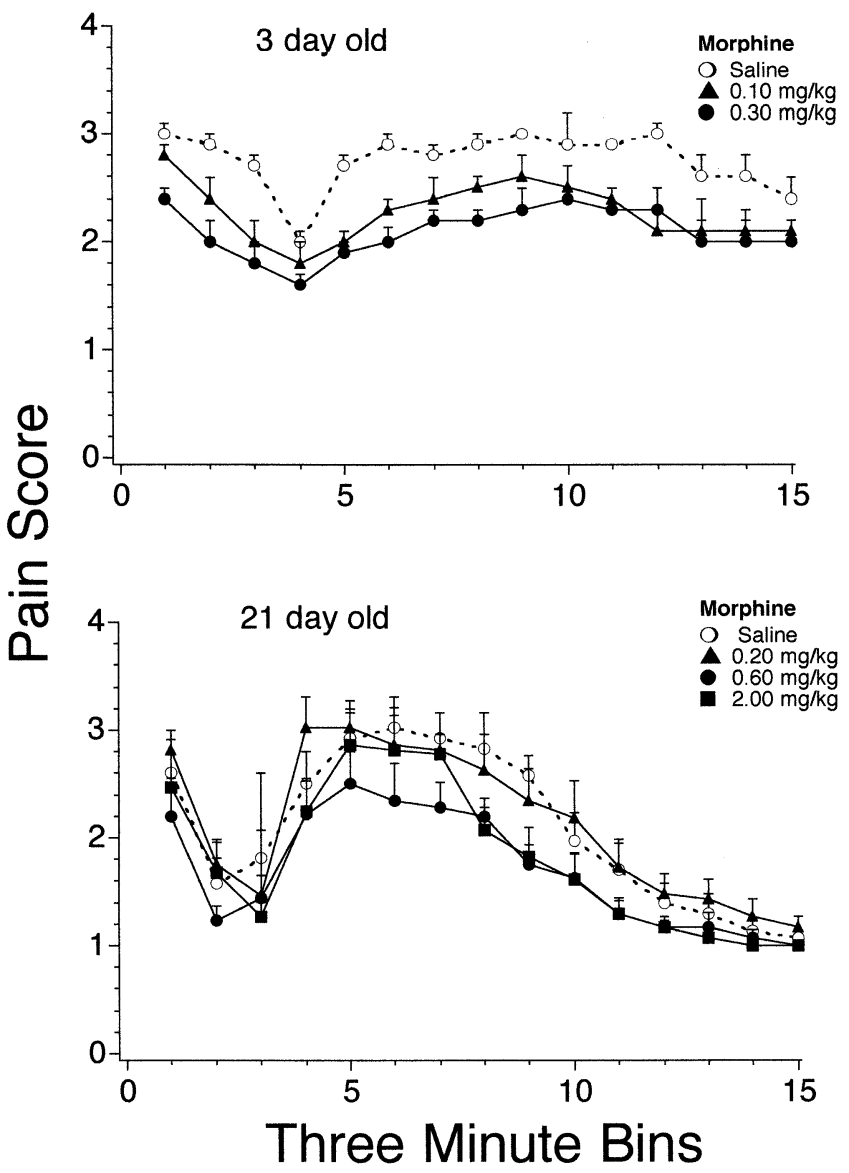

Fig. 2. Mean ( \pm 1 S.E.M.) nociceptive scores across the first 15 threeminute bins in 3-day-old rat pups that received intraperitoneal saline, 0.1 , or $0.3 \mathrm{mg} / \mathrm{kg}$ of morphine and 21-day-old rat pups that received intraperitoneal saline, $0.2,0.6$, or $2.0 \mathrm{mg} / \mathrm{kg}$ of morphine prior to an intraplantar injection of $2 \%$ formalin $(10 \mu \mathrm{l})$. The highest dose in 3-day-old pups was sedating. In both groups, morphine reduced nociceptive scores during phases 1 and 2 . In 3-day-old pups, both low and medium doses showed a significant effect $(P<.01)$, and in 21-day-old pups, medium dose $(P<.05)$ and high dose $(P<.01)$ showed a significant effect.

\subsection{Paw diameter}

One hour after the formalin injection, the dorso-ventral diameter of right and left hindpaw of each pup was measured using calipers accurate to 0.001 in.. The right hindpaw diameter served as a control for the degree of inflammation in the left hindpaw. Data are expressed as diameter of control paw divided by diameter of inflamed paw multiplied by 100 .

\subsection{Immunocytochemistry}

We performed quantitative immunocytochemistry of spinal cords for Fos staining. There is evidence of some correlation between Fos staining and behavioral nociception in infant animals (Yi and Barr, 1995). A reduction in Fos staining of the spinal cord has also been reported with analgesia induced by opioids (Gogas et al., 1996) and
NSAIDs (Buritova et al., 1995). Since the expression of Fos protein in the dorsal horn of the spinal cord peaks at $2 \mathrm{~h}$ after formalin injection, pups were sacrificed at this time and perfused with phosphate buffered saline followed by paraformaldehyde as previously described (Yi and Barr, 1995). Thirty-micron sections of the lumbar enlargement of the spinal cord were stained for Fos protein using the avidinbiotin-peroxidase method. Fos-stained nuclei were counted as described previously (Yi and Barr, 1995). Each section was subdivided into five regions, and all stained nuclei within each region were counted. For each pup, nuclei were counted from five sections, and the mean number of stained nuclei per region was obtained. We did not measure Fos in the morphine group, since previous studies have shown reduction in Fos staining with morphine at both 3 and 21 days of age (Yi and Barr, 1998). We realize that behavioral testing and Fos staining are not isomorphic measures of nociception. However, since the 3-day pups treated with ketorolac did not show any difference in their behavioral scoring, we decided not to examine their spinal cords for Fos staining. Thus, we used Fos expression as a confirmation of the behavioral results in the 21-day-old pups treated with ketorolac, and to identify a reduction of nociceptioninduced Fos expression at the level of the spinal cord.

\subsection{Statistics}

For the behavioral analysis, the minute-by-minute data were averaged into 3 -min bins to decrease the variability. The pain score for each 3-min bin (during the first $45 \mathrm{~min}$ ) was analyzed using two-way analysis of variance (ANOVA) with the 3-min bins $(n=15)$ and drug dose as within-subjects variables. Although pain scores were recorded over $60 \mathrm{~min}$, only the first $45 \mathrm{~min}$ were analyzed since the 21-day-old pups showed no nociceptive behavior in the last $15 \mathrm{~min}$ of

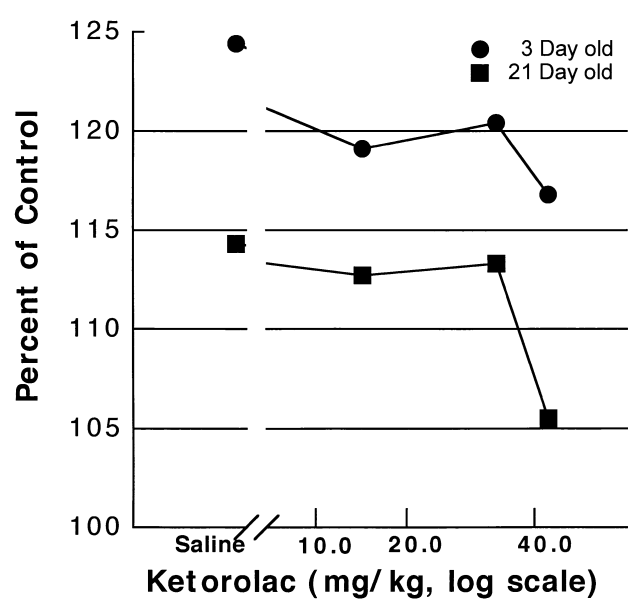

Fig. 3. Comparison of edema in formalin-injected vs. control hindpaw in 21-day-old pups treated with saline, 15,35 , or $45 \mathrm{mg} / \mathrm{kg}$ of ketorolac. Highest dose of ketorolac only at 21 days reduced edema $(P<.05)$. Since this comparison of the treated paw vs. control paw is a percent difference between the two paws, there are no standard error bars. 
the 60-min observation period. For the inflammation assessment, an ANOVA was performed, with the paw diameter and the drug dose as within-subjects variables. For the Fos analysis, a one-way ANOVA was used, with the drug dose as the within-subjects variable. Post hoc analysis was performed for drug-dose effects using the Newman-Keuls test. Statistical tests were considered significant if $P \leq .05$.

\section{Results}

\subsection{Behavioral assessment: pain scores}

\subsubsection{Ketorolac}

At 3 days of age, there was no reduction in pain scores at any dose of ketorolac (Fig. 1). As expected at this age, the scores remained high throughout, with no clear distinction of phases 1 and 2 (Barr, 1998; Guy and Abbott, 1992; Teng
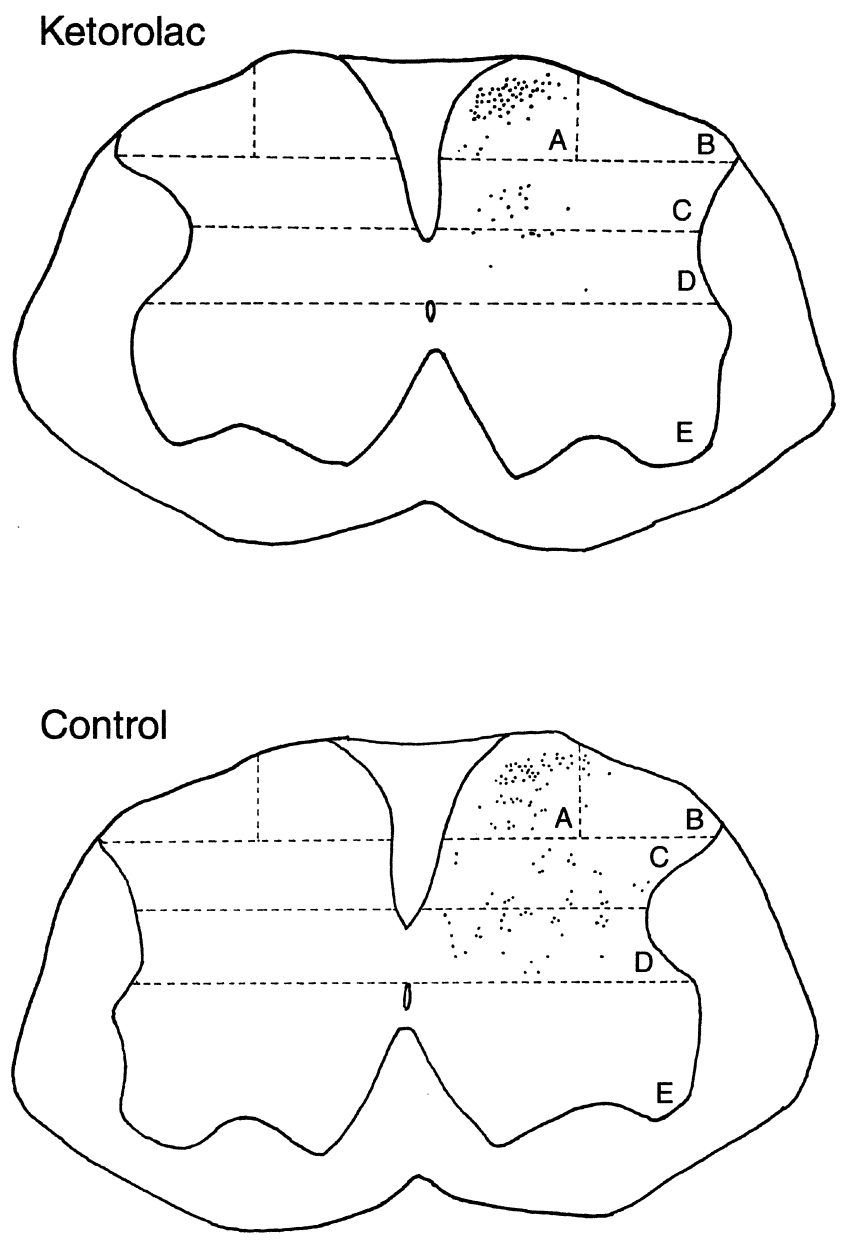

Fig. 4. Camera lucida drawing of Fos expression in the lumbar spinal cord of a representative 21-day-old pup treated with saline (top) or $45 \mathrm{mg} / \mathrm{kg}$ of ketorolac (bottom). Ketorolac-treated animals had fewer Fos-stained nuclei. Regions of the spinal cord that were counted for Fos: areas A and B approximately correspond to the medial and lateral superficial lamina respectively, area $\mathrm{C}$ approximately corresponds to laminae 3 and 4, area D to lamina 5, and area $\mathrm{E}$ to the ventral horn.

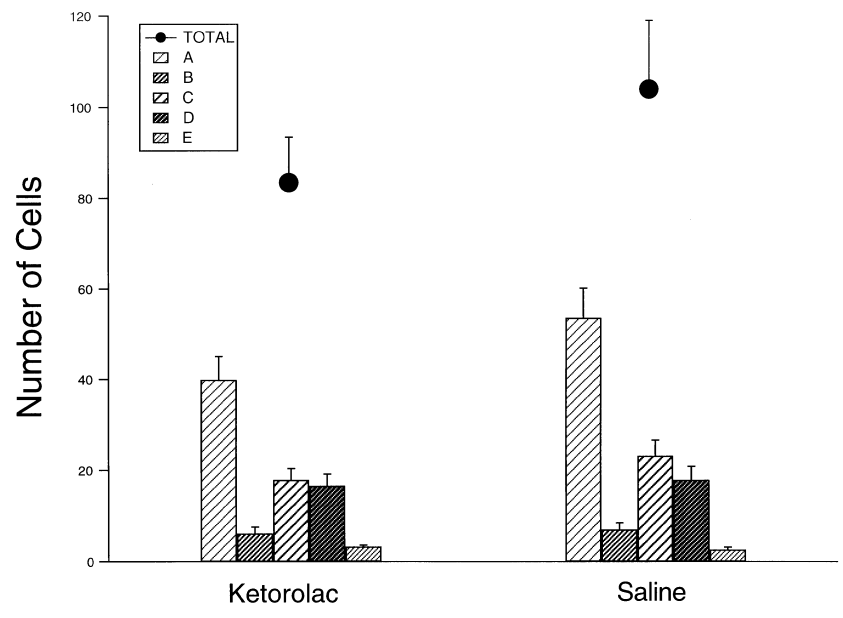

Fig. 5. Number of Fos-stained nuclei in various regions of spinal cord in 21day-old pups treated with saline or $45 \mathrm{mg} / \mathrm{kg}$ of ketorolac. There was a reduction in Fos-stained nuclei in ketorolac-treated animals ( $P=.055)$; most of this reduction was in areas $\mathrm{A}$ and $\mathrm{C}$.

and Abbott, 1998) (Fig. 3). At 21 days of age, a dosedependent reduction in pain scores was seen $\left(F_{3,21}=6.11\right.$, $P<.01)$. On post hoc analysis, a statistically significant difference was seen between saline-treated animals and those treated with $45 \mathrm{mg} / \mathrm{kg}$ (highest dose) ketorolac $(P<.01)$ (Fig. 2). There was no significant interaction between dose and bins, i.e. the dose effect was consistent through all of the bins $\left(F_{42,294}=0.67, P>.05\right)$.

\subsubsection{Morphine}

The 3-day-old pups treated with the highest dose of morphine appeared sedated, and we tested a separate group of pups of the same age for the righting reflex, with morphine $[0.1,0.3$, or $1 \mathrm{mg} / \mathrm{kg}$ to 3 -day-old pups $(n=3 /$ dose $)]$. At the highest dose, the righting reflex was abolished; at the low and medium doses, the righting reflex was preserved and the animals did not appear sedated. Thus, we did not include the high dose data in the analysis. The 3-day-old pups demonstrated a statistically significant, dose-dependent reduction in pain scores $\left(F_{2,14}=24.3, P<.01\right)$ (Fig. 2). There was no significant interaction between dose and bins $\left(F_{28,196}=1.03\right.$, $P>.05)$. Post hoc analysis showed that both doses $(P<.01)$ significantly reduced pain scores relative to the saline vehicle. At 21 days of age, there was a statistically significant reduction in pain scores $\left(F_{3,27}=8.04, P<.01\right)$, with no significant interaction between dose and bins $\left(F_{42,378}=1.02\right.$, $P>.05)$ (Fig. 3). On post hoc analysis, the medium $(0.6 \mathrm{mg} /$ $\mathrm{kg})(P<.01)$ and high $(2 \mathrm{mg} / \mathrm{kg})(P<.05)$ doses of morphine showed a significant reduction in nociceptive responding relative to the vehicle control. There was no difference between the medium and high doses $(P>.05)$.

\subsection{Inflammation: paw diameter}

All animals had edema in their formalin-injected paws, and the injected hindpaw diameters were greater than the 
control hindpaw diameters. At 3 days, there was no reduction in left hindpaw diameters in the ketorolac treated animals compared to controls. At 21 days, animals treated with the highest dose ketorolac had significantly less edema in the left paw, as measured by paw diameter, compared to control animals $(P<.05)$ (Fig. 3). There was no reduction in the diameter of the formalin injected paw in the morphinetreated animals (data not shown).

\subsection{Neural response: Fos}

In the 21-day animals treated with ketorolac, we compared the Fos staining in the spinal cord on the side of the formalin injection between the saline treated group and the high-dose ketorolac group. Since the Fos staining was largely absent in the dorsal horn of the contralateral side, we did not analyze that side. We found a trend towards reduced staining in the ketorolac treated animals $(P=.055)$ (Figs. 4 and 5). The most prominent difference as expected was seen in area $\mathrm{A}$, which corresponds with the medial aspects of Laminae 1 and 2 (Fig. 5).

\section{Discussion}

Ketorolac is an effective analgesic and antiinflammatory drug in the formalin model in adult rats with a range of minimal effective doses between 4.5 and $40 \mathrm{mg} / \mathrm{kg}$ (Randolph and Peters, 1997; Bustamente and Paeile., 1993). In our study, in 21-day-old rats injected with subcutaneous formalin, ketorolac was also effective as an analgesic agent, as seen by the reduction in pain scores on behavioral testing, and as an antiinflammatory agent, as seen by the reduction in paw edema. At this age, ketorolac caused a reduction in pain scores through all the bins, i.e. through phases 1 and 2. In adult rats, ketorolac had an effect only during phase 2 of the formalin test (Randolph and Peters, 1997). Ketorolac-treated rats also showed a trend towards reduced Fos staining in their spinal cords, which is consistent with the behavioral data showing that ketorolac attenuates nociception at this age. The maximal response for analgesia was seen at $45 \mathrm{mg} / \mathrm{kg}$. In adult rats, ketorolac had an analgesic effect at $40 \mathrm{mg} / \mathrm{kg}$, but not at $20 \mathrm{mg} / \mathrm{kg}$ (Bustamente and Paeile., 1993). Thus, the doses that we found effective at 21 days of age were closer to those in the study by Bustamente and Paeile Ketorolac, at the age of 3 days, did not show any significant analgesic or antiinflammatory activity. At this age, we did not see a biphasic response in the pain scores, this is consistent with previous reports (Barr, 1998; Guy and Abbott, 1992; Teng and Abbott, 1998). Whether higher doses of ketorolac would be effective at this age is not known. The role of prostaglandins in inflammatory hyperalgesia in neonatal rats is also not known. Since there is no definite second phase, it is possible that they do not play a major role, and this may be the reason why we did not see a response to ketorolac at this age. Other mediators such as glutamate may play a more significant role in inflammation at the younger ages. Another explanation for ketorolac's inefficacy may be because of different pharmacokinetics of ketorolac in neonatal rats, but we are not aware of any such data.

Morphine produced significant reduction in pain scores in 3- and 21-day-old rats. There was no effect on inflammation as treatment with morphine did not reduce paw edema. This was not unexpected, since morphine has no antiinflammatory activity. We recognize that it is difficult to compare the two ages because of the different doses used, but morphine is known to be more potent in younger rat pups, part of the reason being their immature blood-brain barrier. At 0.1 and $0.3 \mathrm{mg} / \mathrm{kg}$, there was significant reduction in pain scores without apparent sedation across the 45min session. The control animals in the 3-day morphine group had higher scores than the control group in the 3-day ketorolac animals, and we cannot explain this difference other than interlitter variations. Although the scores of the morphine-treated animals are similar to the scores of the control animals in the ketorolac group, we think that the effect of morphine is real, since each experiment compared drug doses between littermates, thus, minimizing interlitter variations. In 21-day-old rats, both the medium and high doses produced a statistically significant reduction in pain scores in both phases of the test. In adult rats also, morphine has been shown to reduce pain scores in both phases of the test (Randolph and Peters, 1997).

On the basis of brain growth rate, neonatal rats are equivalent to human preterm infants of 26 weeks gestational age, 7-day-old rat pups are equivalent to term human neonates and 21-day-old rats are equivalent to young children (Dobbing, 1981). This study demonstrates that ketorolac is an active analgesic and antiinflammatory drug in 21-day-old rats, but not in 3-day-old rats; whether it is effective at intermediate ages remains to be seen. We also confirmed that morphine is a potent analgesic agent in 3- and 21-day-old rats. Though the analgesic effects of both ketorolac and morphine were modest in 21-day-old pups, morphine is clinically a potent analgesic in human neonates. This study may have some clinical relevance in that ketorolac may not be effective in human premature infants, but may be effective at an older age, i.e. term or during infancy, and morphine or other opiates may be the analgesic drugs of choice in premature babies.

\section{References}

Barr GA. Maturation of the biphasic behavioral and heart rate response in the formalin test. Pharmacol, Biochem Behav 1998;60:329-35.

Buritova J, Honoré P, Chapman V, Besson J-M. Carrageenan oedema and spinal Fos-L1 neurons by systemic diclofenac in the rat. NeuroReport $1995 ; 6: 1257-60$

Chiaretti A, Simeone E, Langer A, Butera G, Piastra M, Tortorolo L, Poliori G. Analgesic efficacy of ketorolac and fentanyl in pediatric intensive care. Pediatr Med Chir 1997;19:419-24. 
Dobbing J. Later development of the brain and its vulnerability. In: Davis J, Dobbing J, editors. Scientific foundations of paediatrics, vol. 2. 1981. pp. 744-59. London: William Heinemann.

Gillis JC, Brogden RN. Ketorolac. A reappraisal of its pharmacodynamic and pharmacokinetic properties and therapeutic use in pain management. Drugs 1997;53:139-88.

Gogas KR, Cho HJ, Botchkina GI, Levine JD, Basbaum AI. Inhibition of noxious stimulus-evoked pain behaviors and neuronal fos-like immunoreactivity in the spinal cord of the rat by supraspinal morphine. Pain 1996;65:9-15.

Gonzalez A, Smith DP. Minimizing hospital length of stay in children undergoing ureteroneocystostomy. Urology 1998;52:501-4.

Granados-Soto V, Flores-Murrieta FJ, Castaneda-Hernandez G, Hong E, Lopez-Munoz FJ. Comparison of analgesic effects of ketorolac, morphine and aspirin in the rat. Proc West Pharmacol Soc 1993;36:245-8.

Guy ER, Abbott FV. The behavioral response to formalin in preweanling rats. Pain 1992;51:81-90.

Jett M-F, Ramesha CS, Brown CD, Chiu S, Emmett C, Voronin T, Sun T, O'Yang C, Hunter JC, Eglen RM, Johnson RM. Characterization of the analgesic and anti-inflammatory activities of ketorolac and its enantiomers in the rat. J Pharmacol Exp Ther 1999;288:1288-97.

Larkin GL, Peacock WF, Pear SM, Blair GA, D'Amico F. Efficacy of ketorolac tromethamine versus meperidine in the ED treatment of acute renal colic. Am J Emerg Med 1999;17:6-10.

Mather SJ, Peutrell JM. Postoperative morphine requirements, nausea and vomiting following anaesthesia for tonsillectomy. Comparison of intravenous morphine and non-opioid analgesic techniques. Paediatr Anaesth 1995;5:185-8.

Maves TJ, Pechman PS, Meller ST, Gebhart GF. Ketorolac potentiates morphine antinociception during visceral nociception in the rat. Anesthesiology 1994;80:1094-101.

Picard P, Bazin JE, Conio N, Ruiz F, Schoffler P. Ketorolac potentiates morphine in postoperative patient-controlled analgesia. Pain 1997;73: $401-6$.

Purday JP, Reichert CC, Merrick M. Comparative effects of three doses of intravenous ketorolac or morphine on emesis and analgesia for restorative dental surgery in children. Can J Anesth 1996;43:221-5.

Randolph BC, Peters MA. Analgesic effectiveness of ketorolac compared to meperidine in the rat formalin test. Anesth Prog 1997;44:11-6.

Rooks WH. The pharmacologic activity of ketorolac tromethamine. Pharmacotherapy 1990;10:30S-2S.

Rooks WH, Maloney PJ, Shott LD, Shuker ME, Sevelius H, Strosberg AM, Tanenbaum L, Tomolinos AJ, Wallach MB, Waterbury D, Yee JP. The analgesic and anti-inflammatory profile of ketorolac and its tromethamine salt. Drugs Exp Clin Res 1985;11(8):479-92.

Shende D, Das K. Comparative effects of intravenous ketorolac and pethidine on perioperative analgesia and postoperative nausea and vomiting (PONV) for paediatric strabismus surgery. Acta Anesthesiol Scand 1999;43:265-9.

Teng CJ, Abbott FV. The formalin test: a dose-response analysis at three developmental stages. Pain 1998;76:337-47.

Uphouse LA, Welch SP, Ward CR, Ellis EF, Embrey JP. Antinociceptive activity of intrathecal ketorolac is blocked by the $\kappa$-opioid receptor antagonist, nor-binaltorphimine. Eur J Pharmacol 1993;21(242):53-8.

Yi DK, Barr GA. The induction of Fos-like immunoreactivity by noxious thermal, mechanical and chemical stimuli in the lumbar spinal cord of infant rats. Pain 1995;60:257-65.

Yi DK, Barr GA. The ontogeny of nociception and antinociception in the fetal and infant rat spinal cord using fos protein as a marker. Unpublished $\mathrm{PhD}$ thesis, 1998.

Zhang Y, Shaffer A, Portanova J, Seibert K, Isakson PC. Inhibition of cyclooxygenase-2 rapidly reverses inflammatory hyperalgesia and prostaglandin E2 production. J Pharmacol Exp Ther 1997;283:1069-75. 